\title{
Rapid Regulation of Alternative Splicing in Response to Environmental Stresses
}

\section{OPEN ACCESS}

Edited by:

Kranthi Kiran Mandadi,

Texas A\&M University, United States

Reviewed by:

Sureshkumar Balasubramanian,

Monash University, Australia

Xiangjia Min,

Youngstown State University,

United States

*Correspondence:

Peng Liu

pliu@danforthcenter.org

Kang Yan

kangyan@sdau.edu.cn

tThese authors have contributed equally to this work

Specialty section:

This article was submitted to Plant Physiology,

a section of the journal

Frontiers in Plant Science

Received: 09 December 2021

Accepted: 25 January 2022

Published: 04 March 2022

Citation:

Liu X-X, Guo Q-H, Xu W-B, Liu P and Yan K (2022) Rapid Regulation of Alternative Splicing in Response

to Environmental Stresses.

Front. Plant Sci. 13:832177.

doi: 10.3389/fp/s.2022.832177
Xiao-Xiao Liu't, Qian-Huan Guo ${ }^{1 \dagger}$, Wei-Bo Xu', Peng Liü ${ }^{2 *}$ and Kang Yan ${ }^{1 *}$

${ }^{1}$ State Key Laboratory of Crop Biology, College of Life Sciences, Shandong Agricultural University, Tai'an, China, ${ }^{2}$ Donald

Danforth Plant Science Center, St. Louis, MO, United States

Plants overcome the changing environmental conditions through diverse strategies and complex regulations. In addition to direct regulation of gene transcription, alternative splicing (AS) also acts as a crucial regulatory mechanism to cope with various stresses. Generating from the same pre-mRNA, AS events allow rapid adjustment of the abundance and function of key stress-response components. Mounting evidence has indicated the close link between AS and plant stress response. However, the mechanisms on how environmental stresses trigger AS are far from understood. The advancing high-throughput sequencing technologies have been providing useful information, whereas genetic approaches have also yielded remarkable phenotypic evidence for AS control of stress responses. It is important to study how stresses trigger AS events for both fundamental science and applications. We review current understanding of stress-responsive AS in plants and discuss research challenges for the near future, including regulation of splicing factors, epigenetic modifications, the shared targets of splice isoforms, and the stress-adjusting ratios between splicing variants.

Keywords: alternative splicing, environmental stress, post-transcriptional regulation, splicing factor, epigenetic control

\section{INTRODUCTION}

Since RNA splicing was initially discovered in 1977, this process of removing introns from premRNA has been observed in most eukaryotic cells (Berget et al., 1977). The accuracy of RNA splicing is crucial for the synthesis of functional proteins. Although there are multiple splicing mechanisms, canonical splicing which is catalyzed by spliceosome accounts for the majority. The selection of splice sites is not only determined by core spliceosomal components but also regulated by a number of spliceosome-associated RNA binding factors, predominantly serine/arginine-rich (SR) proteins and other splicing factors (Laloum et al., 2018).

In response to changing environmental conditions, alternative mature transcripts from the same pre-mRNA can be generated rapidly by choosing different splicing sites (Laloum et al., 2018). AS greatly enhances the coding capacity of a genome and expands the proteome, regulating up to $95 \%$ of human and 70\% of plant multi-exon genes (Pan et al., 2008; Zhang et al., 2010, 2017; Marquez et al., 2012). Intron retention (IR) is predominant in plants and exon skipping (ES) is the most frequent AS event in mammals (Gupta et al., 2004; Wang and Brendel, 2006). Notably, RNA-seq data have been confirming previous indications that abiotic stress markedly enhances AS events in plants (Laloum et al., 2018). 
According to a recent study, different stresses rarely induce overlapped AS events in plants, suggesting an environmental specificity of AS regulations (Punzo et al., 2020). The selection of alternative splice sites was enhanced for more than 6,000 genes in Arabidopsis under high salinity (Feng et al., 2015). A large number of differential AS events in maize leaves were found when exposed to heat stress, and more than half of them are ES and IR (Li Z. et al., 2021). During the sharp cooling treatment on tea plants, the numbers of AS events were also significantly increased (Li et al., 2020). These observations have indicated that pre-mRNA splicing may have a strong bearing on stress response in plants. AS patterns can be altered directly by splicing factors or epigenetic changes, here we will review current studies on how stresses control AS through these mechanisms in plants. We will also discuss the consequences of AS and its influences on plant stress responses (Table 1).

\section{DIRECT REGULATION BY SPLICING FACTORS}

Alternative splicing is most commonly controlled by splicing factors, and AS regulation studies have been primarily focusing on key RNA sequence elements and their associated regulators (Luco et al., 2011). Plant stress-responsive genes are particularly prone to generating multiple transcripts in response to different environmental stresses (Ner-Gaon et al., 2004). However, the conserved and specific stress-responsive RNA sequence elements were not found among plant species, and AS events from different genes revealed completely diversified splicing recognition sites (Laloum et al., 2018). These suggest that the splicing mechanism in response to environmental stresses is more dependent on a variety of splicing factors in plants.

Splicing factor genes usually show a quick response to environmental stresses at transcriptional levels (Laloum et al., 2018). For example, the SR34b gene is upregulated by cadmium (Cd), and controls the plant tolerance to $\mathrm{Cd}$ toxicity in Arabidopsis (Zhang et al., 2014). Two plant-specific SC35-Like (SCL) SR genes are downregulated upon exposure to exogenous ABA treatment in Arabidopsis (Cruz et al., 2014). The U5 snRNP-associated splicing factor, STABILIZED1 (STA1) gene is upregulated in response to cold stress, and the phenotypes of sta1-1 plants under cold stress is severe (Lee et al., 2006). The transcriptional and protein levels of the Ser/Arg-rich splicing factor SR45, SR30, and SR34, and the nuclear ribonucleic protein $\mathrm{U} 1 \mathrm{~A}$ accumulate under high temperature in Vitis vinifera (Jiang et al., 2017). Also, the expression levels of splicing factor PRP18 shows large increases under drought conditions in maize (Thatcher et al., 2016). Some splicing factors themselves undergo AS events under stress, like SR45a, in which two splice isoforms showed strong induction by salt treatment (Li Y. et al., 2021).

The change of splicing factor transcriptional expressions usually leads to the altered AS patterns of downstream stressresponsive genes (Figure 1A). Ski-interacting protein (SKIP) is a salt-responsive splicing factor, which mediates the AS of many genes in the recognition and cleavage of $5^{\prime}$ donor and $3^{\prime}$ acceptor sites (Feng et al., 2015). Salt stress changes SKIP expression levels and decreases the ability of the spliceosome to accurately recognize splice sites (Feng et al., 2015). Splicing factor Sm protein E1 (SME1) ensures the appropriate splicing of a high number of pre-mRNAs in maintaining the levels of selected cold-responsive functional transcripts (Huertas et al., 2019). SME1 shows a cold response expression pattern, and the mutant phenotype displays that SME1 functions as a negative regulator of the cold acclimation process by regulating splicing events (Huertas et al., 2019). RNA-directed DNA methylation 16 (RDM16) encodes an $\mathrm{ABA}$ responsive pre-mRNA-splicing factor 3(PRP3), which is involved in pre-mRNA splicing. And RNA-seq data identified 308 IR events changed in $r d m 16$ mutant (Huang et al., 2013).

In addition to the transcriptional regulations, splicing factors are also modified at post-translational levels. Most of the nuclear SR proteins are phosphorylated on their RS domain, and the phosphorylation status of SR proteins is highly related to their functions in spliceosome assembly and subcellular localization (Jeong, 2017). The phosphorylation of glycine-rich RNA binding protein7 (GRP7) enhances its mRNA binding ability and its association with spliceosome component U1-70K to change dynamic AS (Wang et al., 2020). The alkalinization of FACTOR 1 (RALF1) and FERONIA (FER) triggers rapid and massive AS events by interacting with and phosphorylating GRP7 in Arabidopsis (Wang et al., 2020).

\section{EPIGENETIC CONTROL}

Epigenetic markers, like chromatin modification and DNA methylation have also been found to be associated with AS regulations. Dramatic epigenetic changes play key roles in celland condition-dependent AS regulation in animals (Ibtissam et al., 2019). In plants, the role of epigenetic control in regulating AS under stress is emerging ( $\mathrm{Hu}$ J. et al., 2019). Recent data have identified a strong relationship between chromatin changes and AS control. A potential epigenetic control of AS is through pol II (Figure 1B). The pol II initiation and elongation speed mediate the splicing processing of pre-mRNAs to generate AS transcripts in plants (Ibtissam et al., 2019). Greater pol II processivity is associated with a more open chromatin structure, which favors pol II elongation (Petrillo et al., 2014; Godoy Herz et al., 2019). In Arabidopsis and rice, the chromatin structure was more open in retained introns (Fahad et al., 2018). The open chromatin architecture enhanced pol II elongation rate, which led to skipping of splice sites (Fahad et al., 2018). In addition, pol II elongation speed was also found to be slower in exons than introns in Arabidopsis (Ibtissam et al., 2019). Interestingly, pol II elongation speed is faster under light conditions than in darkness, leading to an ES (Godoy Herz et al., 2019). DNA methylation is also associated with chromatin remodeling to regulate plant AS patterns. In rice, the widespread differences of splicing variants were found in CG methyltransferase mutant OsMet1-2 lines (Wang et al., 2016). Also, CG methylation was higher in AS-related introns than constitutive introns (Wang et al., 2018). 
TABLE 1 | A summary of stress-responsive AS genes.

\begin{tabular}{|c|c|c|c|c|c|}
\hline Gene & Specie & Stress responses & Function & Regulation types of AS* & References \\
\hline$S R 34 b$ & Arabidopsis & Cadmium & Splicing factors & $A$ & Zhang et al., 2014 \\
\hline SR45 & Vitis vinifera & Heat & Splicing factors & A & Jiang et al., 2017 \\
\hline SR30 & Vitis vinifera & Heat & Splicing factors & A & Jiang et al., 2017 \\
\hline SR34 & Vitis vinifera & Heat & Splicing factors & A & Jiang et al., 2017 \\
\hline$S C L$ & Arabidopsis & $\mathrm{ABA}$ & Splicing factors & $A$ & Cruz et al., 2014 \\
\hline SR45a & maize & Heat & Splicing factors & A & Li Z. et al., 2021 \\
\hline STA1 & Arabidopsis & Cold & Splicing factor & A & Lee et al., 2006 \\
\hline SKIP & Arabidopsis & Salt & Splicing factor & A & Feng et al., 2015 \\
\hline SME1 & Arabidopsis & Cold & Splicing factor Sm protein & A & Huertas et al., 2019 \\
\hline$R D M 16$ & Arabidopsis & $\mathrm{ABA}$ & ABA responsive splicing factor & A & Huang et al., 2013 \\
\hline PRP18 & maize & Drought & Splicing factor & A & Thatcher et al., 2016 \\
\hline GRP7 & Arabidopsis & Salt & Glycine-rich RNA binding protein & A & Wang et al., 2020 \\
\hline SKB1 & Arabidopsis & Salt, ABA & Shk1 kinase binding protein & $\mathrm{B}$ & Zhang et al., 2011 \\
\hline MET1-2 & Rice & Cadmium & CG methyltransferase & $\mathrm{B}$ & Wang et al., 2015 \\
\hline FLC & Arabidopsis & Salt & Transcription factor & $\mathrm{B}$ & Zhang et al., 2011 \\
\hline HAB1 & Arabidopsis & ABA & Type 2C protein phosphatase & C D & Wang et al., 2015 \\
\hline CIPK3 & Arabidopsis & ABA, Drought & Serine-threonine protein kinase & C & Sanyal et al., 2017 \\
\hline HsfA2 & Arabidopsis & Heat & Transcription factor & C & Liu et al., 2013 \\
\hline HSFA & Tomato, Lily & Heat & Transcription factor & C & Hu Y. et al., 2019; Wu et al., 2019 \\
\hline IDD14 & Arabidopsis & Cold & Inderminate domain & C & Seo et al., 2011 \\
\hline SR45a & Arabidopsis & Salt & SR like protein & C D & Li Y. et al., 2021 \\
\hline SRAS1 & Arabidopsis & Salt & Salt-responsive AS gene & CD & Zhou et al., 2021 \\
\hline CBP2O & Arabidopsis & Salt & Cap-binding protein & C & Li Y. et al., 2021 \\
\hline CSN5A & Arabidopsis & ABA, Salt & COP9 signalosome & C & Zhou et al., 2021 \\
\hline$A B / 3$ & Arabidopsis & ABA & Transcription factor & $\mathrm{D}$ & Sugliani et al., 2010 \\
\hline$R B M 25$ & Arabidopsis & $\mathrm{ABA}$ & RNA-binding protein & D & Cheng et al., 2017 \\
\hline VVPMA1 & Vitis vinifera & Salt & $\mathrm{PM} \mathrm{H}^{+}$-ATPase genes & D & Han et al., 2017 \\
\hline LUC7 & Arabidopsis & Cold & Lethal unless CBC7 & D & Marcella et al., 2018 \\
\hline FLM & Arabidopsis & Heat & MADS domain protein & D & Chang et al., 2021 \\
\hline CML21 & Vitis vinifera & Cold & Calmodulin-Like Gene & $\mathrm{D}$ & Aleynova et al., 2020 \\
\hline
\end{tabular}

*Different types of AS are illustrated in the Figure 1.

A close relationship between abundant epigenetic modifications and splicing variation has been revealed under different growth and stress conditions. Under salt stress, PRMT5 (protein arginine methyltransferase 5) methyltransferase (also known as SKB1) increases H4R3sme2 (histone 4 arginine 3 symmetric demethylation) levels in Arabidopsis, suggesting SKB1 disassociation from chromatin results in a reduction in the cellular levels of H4R3sme2, resulting in the induction of FLOWERING LOCUS C (FLC) and salt stress-responsive genes (Zhang et al., 2011). PRMT5 also alters AS in the core clock gene PSEUDO RESPONSE REGULATOR 9 (PRR9) and influences clock functioning in Arabidopsis (Sanchez et al., 2010). Evidence in rice indicates that histone H3K36-specific methyltransferase (SDG725) regulates IR events in many genes (Wei et al., 2018). In Arabidopsis, temperature-induced differentially spliced genes are enriched in H3K36me3 marks, while depletion of H3k36me3 marks has the opposite effect to temperature-induced AS (Pajoro et al., 2017).

\section{SHARED TARGET BINDING AND REGULATING}

In consequence of AS, novel protein products may be generated and involved in the plant stress responses. AS events often introduce premature stop codons, generating truncated isoforms. Interestingly, these truncated isoforms often keep the ability of interacting with the same target with full-length protein, while some ability is lost because of missing key domain (Figure 1C). In the $\mathrm{ABA}$ signaling pathway, the Group A protein type $2 \mathrm{C}$ phosphatases (PP2C) HAB1 undergoes ABA-controlled AS to produce two splice variants, which encode HAB1.1 and HAB1.2. Both of them interact with subclass III SNF1-related protein kinases SnRK2.6 (OST1) in both cytoplasm and nucleus (Wang et al., 2015). Another example is the CIPK3 splice variants induced by $\mathrm{ABA}$ and drought treatment. Five CIPK3 protein variants (CIPK3.1, CIPK3.2, CIPK3.3, CIPK3.4, and CIPK3.5) have been generated. Although having different preferences on their upstream CBL interactors, these proteins do not lose the binding ability to their target (Sanyal et al., 2017). 


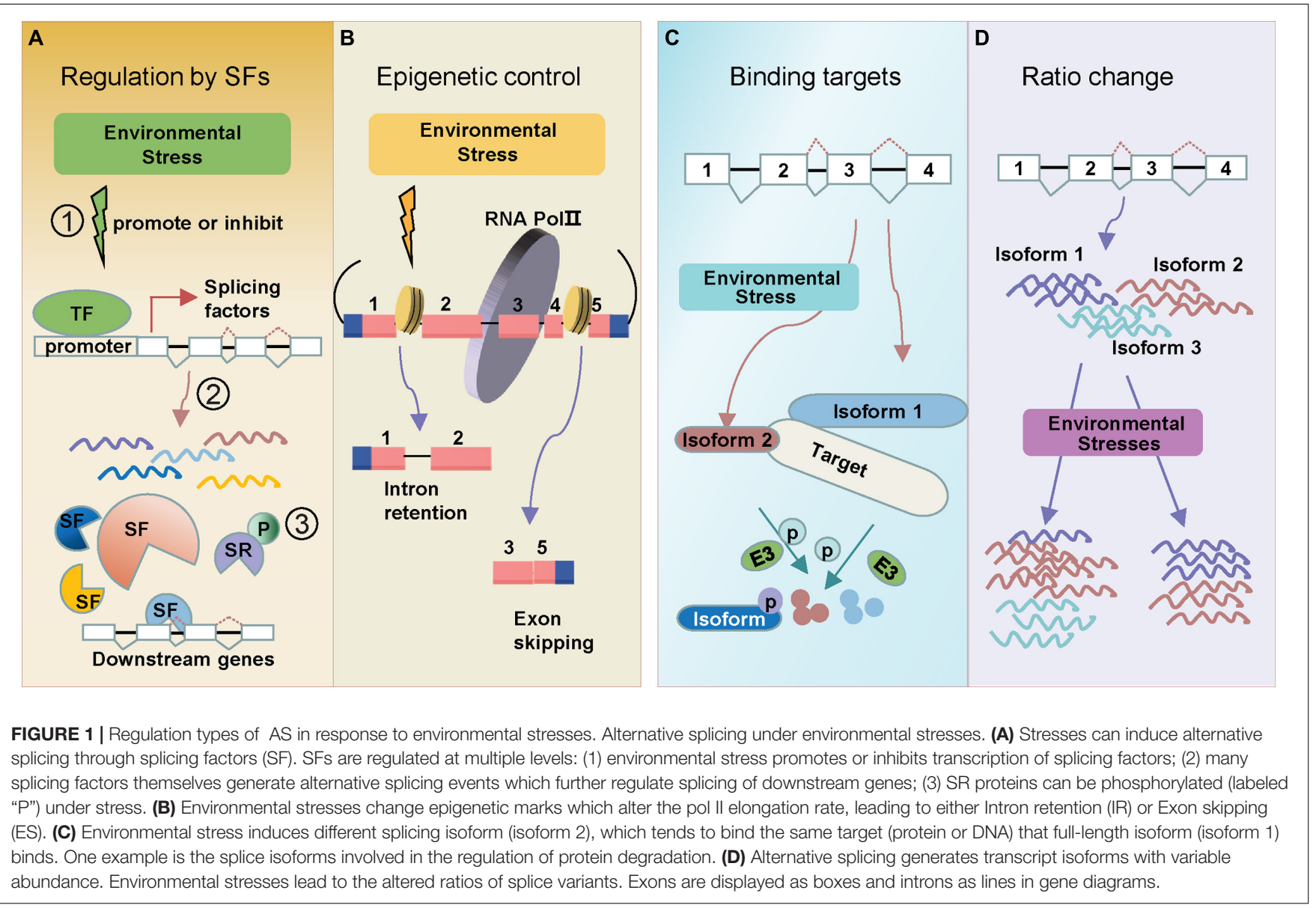

A key transcription factor Heat shock transcription factorA2 (HsfA2) generate a new intron-retained splice variant (designated HsfA2-III) in Arabidopsis seedlings (Liu et al., 2013). This truncated isoform still has DNA-binding ability, but the loss of C-terminal activation domain (CTAD) of HsfA2 might lead to the defect of transactivation activity and failure in functioning as a transcription factor (Liu et al., 2013). Similar AS events of HSFA also occur in Tomato and Lily (Hu Y. et al., 2019; Wu et al., 2019). The generation of HSFA splice variants might result in a genetic buffering to tolerate the negative effects of long-term Heat stress on plants (Hu J. et al., 2019). Another temperature-dependent case is the INDERMINATE DOMAIN 14 (IDD14). An alternatively spliced IDD14 form (IDD14 $\beta$ ), which is generated under cold conditions, lacks functional DNA binding domain but is able to form heterodimers with the functional IDD14 form (IDD14 $\alpha$ ) (Seo et al., 2011).

Two splicing variants of serine/arginine-rich (SR)-like protein (SR45a) were also identified under salt stress, full-length SR45a1a and the truncated isoform SR45a-1b. The full-length SR45a1a works as a splicing factor while the truncated isoform SR45a-1b does not (Li Y. et al., 2021). However, the capbinding complex subunit cap-binding protein 20 (CBP20) indeed physically interacts with both SR45a-1a and SR45a1b. SR45a-1b mediates salt-stress signal transduction pathways through promoting the association of SR45a-1a with CBP20
(Li Y. et al., 2021). Another example is the Salt-Responsive Alternatively Spliced gene 1 (SRAS1), encoding a RING-Type E3 ligase. It can generate two splicing variants: SRAS1.1 and SRAS1.2, which exhibit opposing responses to salt stress (Zhou et al., 2021). The full-length SRAS1.1 targets and promotes the degradation of CSN5A, while SRAS1.2 protects CSN5A by competing with SRAS1.1 on the same binding site (Zhou et al., 2021).

\section{ADJUSTMENT OF SPLICING VARIANT RATIOS}

In response to environmental changes, new splicing variants might emerge from stress-responsive genes (Laloum et al., 2018). Simultaneously, the expression levels of original splicing variants might also alter, resulting in an adjustment of splicing variant ratios (Figure 1D). These changes may be essential for plants to adapt to environmental challenges. Following up the above discussion on HAB1, expression analysis showed that the HAB1.2/HAB1.1 ratio greatly differed at the germination stage with or without $\mathrm{ABA}$ treatment (Wang et al., 2015). Under drought treatment, the ratios of HAB1.2/HAB1.1 increased at $0.5 \mathrm{~h}$ and then decreased as time went on in the WT (Cheng et al., 2017). RNA-binding 
protein 25 (RBM25) acts upstream of HAB1, a conserved ABAinduced splicing factor, affecting the ratio of HAB1.2/HAB1.1 to modulate plant response to drought stress (Cheng et al., 2017). There are also some other ratio shifts of splicing variants responding to ABA. Splicing factors suppressor of $\mathrm{ABI} 3$ (SUA) suppresses splicing of the cryptic ABSCISIC ACID INSENSITIVE3 (ABI3) intron and thereby influences the ratio between $\mathrm{ABI} 3-\alpha$ and $\mathrm{ABI} 3-\beta$ transcripts, which then could represent a system to finetune seed maturation (Sugliani et al., 2010).

Similar phenomena were also observed under salt stress. Salt stress triggered the ratio changing of SR45a (SR45a-1a and SR45a-1b) and SRAS1 (SRAS1.1 and SRAS1.2) (Li Y. et al., 2021; Zhou et al., 2021). Another interesting example is the AS events of Vitis vinifera PM H${ }^{+}$-ATPase genes 1 (VvPMA1). The ratio changing of VvPMA1 was discovered under salt conditions (Han et al., 2017). Subtle changes in the ratio of VvPMA1 $\alpha$ and VvPMA1 $\beta$ likely have profound effects on $\mathrm{PM} \mathrm{H}^{+}$-ATPase activity in grape root under salinity (Han et al., 2017).

Besides ABA and salt, extreme temperature also has a dramatic effect on the splice patterns of many genes. Heat and cold stress alter the ratios of splice isoforms for many SR family members, such as SR34b, RS41, and SR30 (Palusa et al., 2007). One additional splice form (isoform 9) of $S R 34 b$ appeared in heat-treated seedlings, whereas some isoforms (3, 6,7 , and 8) were transcriptionally reduced. The ratios of $S R 34 b$ transcripts altered in seedlings with heat or cold treatment (Palusa et al., 2007). The levels of all four splice variants of the Grapevine Calmodulin-Like Gene (VaCML21) were highly induced in response to cold stress (Aleynova et al., 2020). The ratios between transcript variants are changing with or without cold stress (Aleynova et al., 2020). LETHAL UNLESS CBC7 (LUC7) proteins specifically promote a subset of terminal introns splicing in response to cold stress, leading to the ratio changing of splicing variants of cold responsive gene (Marcella et al., 2018).

In Arabidopsis, FLOWERING LOCUS M (FLM) undergoes AS and this temperature-dependent AS leads to a differential accumulation of the FLM- $\beta$ and FLM- $\delta$ transcripts (Lee et al., 2013). Two main splicing variants compete for interaction with the floral repressor SVP to control temperature-dependent flowering (Posé et al., 2013). FLM- $\beta$ was the prevalent splice variant at $16{ }^{\circ} \mathrm{C}$, whereas FLM- $\delta$ dominated at $27{ }^{\circ} \mathrm{C}$. The splice variant ratios of FLM are regulated in response to low and high temperature coupled with nonsense-mediated mRNA decay pathway (Sureshkumar et al., 2016). Also, the splicing events of FLM are mediated by another splicing factor 1 (AtSF1) during its signaling pathway (Kim et al., 2020). AtSF1 acting in $3^{\prime}$ splice-site recognition is responsible for ambient temperature-dependent AS of FLM pre-mRNA, resulting in the temperature-dependent production of functional FLM- $\beta$ transcripts (Kim et al., 2020). The cyclin-dependent kinase G2 (CDKG2), together with its cognate cyclin, CYCLYN L1 (CYCL1) affects the AS of FLM, balancing the levels of FLM$\beta$ and FLM- $\delta$ across the ambient temperature range (Nibau et al., 2020). Both the level and splicing pattern of FLM transcripts are affected by RBP45d and PRP39a, which facilitate temperature-induced AS of FLM to induce flowering at higher temperature (Chang et al., 2021).

\section{CONCLUSION AND FUTURE PROSPECTS}

Evidence has indicated that AS, acting as a crucial regulatory mechanism in response to various stresses, is fast and efficient, and this may have an evolutionary advantage for plants to survive under rapidly changing environments. Although splice variants participate in different pathways, they do have something in common: (1) alternatively spliced transcripts tend to encode truncated proteins that interact with the same targets; and (2) the ratios of splice variants are critical in the regulation of stress response pathways. A key question is how stress signals control splicing factors and lead to AS? Previous studies have shed some light. The reversible phosphorylation might be crucial in the regulation of splicing factor activity. Epigenetics is also associated with AS, and histone markers can alter the pol II elongation speed leading to either ES or IR. More in-depth studies will be necessary to address the upstream regulatory pathways of AS. Another question is how stressinduced AS helps plants to adapt to environmental challenges. More case-by-case studies will be needed to better understand the whole picture.

In addition, CRISPR/Cas9 technology has given us power to engineer the splicing patterns of trait genes for improving crops. The identification of splice variants and function characterization are very helpful for developing new gene editing approaches. Take the FLM variants for example, native FLM genomic locus can be directly edited by deleting exon 2 or 3 to generate splice variants, and the generated germlines display different flowering phenotypes (Capovilla et al., 2017). Instead of deleting entire exons, we could directly modify splice sites in crop genomes to generate desired transcript variants using CRISPR/Cas9 based base editor. The CRISPR/dCas9 could also be coupled with methylation/demethylation enzymes to modulate splicing outcomes through mediating pol II speed. Future studies on stress triggered AS will be of great value in understating the mechanisms of regulation of AS, and in improving crop adaptations to extreme environmental conditions.

\section{AUTHOR CONTRIBUTIONS}

All authors listed have made a substantial, direct, and intellectual contribution to the work, and approved it for publication.

\section{FUNDING}

This research support from the Natural Science Foundation of Shandong Province (Grant ZR2021QC126) and National Natural Science Foundation (Grant Nos. 31870234, 31972357, and 32071931) in China is gratefully acknowledged. 


\section{REFERENCES}

Aleynova, O. A., Kiselev, K. V., Ogneva, Z. V., and Dubrovina, A. S. (2020). The Grapevine Calmodulin-Like Protein Gene CML21 Is Regulated by Alternative Splicing and Involved in Abiotic Stress Response. Int. J. Mol. Sci. 21:7939. doi: 10.3390/ijms21217939

Berget, S. M., Moore, C. M., and Sharp, P. A. (1977). Spliced Segments at the 5' Terminus of Adenovirus 2 Late mRNA. Proc. Natl. Acad. Sci. 74, 3171-3175. doi: $10.1073 /$ pnas.74.8.3171

Capovilla, G., Symeonidi, E., Wu, R., and Schmid, M. (2017). Contribution of major FLM isoforms to temperature-dependent flowering in Arabidopsis thaliana. J. Exp. Bot. 68, 5117-5127. doi: 10.1093/jxb/erx328

Chang, P., Hsieh, H., and Tu, S. (2021). The U1 snRNP component RBP45d regulates temperature-responsive flowering in Arabidopsis. Plant Cell [preprint]. doi: 10.1101/2021.06.14.448173.

Cheng, C., Wang, Z., and Yuan, B. (2017). RBM25 Mediates Abiotic Responses in Plants. Front. Plant Sci. 2017:292. doi: 10.3389/fpls.2017.00292

Cruz, T., Carvalho, R. F., Richardson, D. N., and Duque, P. (2014). Abscisic Acid (ABA) Regulation of Arabidopsis SR Protein Gene Expression. Int. J. Mol. Sci. 15, 17541-17564. doi: 10.3390/ijms151017541

Fahad, U., Michael, H., Reddy, A., and Asa, B. H. (2018). Exploring the relationship between intron retention and chromatin accessibility in plants. BMC Genom. 19:21. doi: 10.1186/s12864-017-4393-Z

Feng, J., Li, J., Gao, Z., Lu, Y., Yu, J., Zheng, Q., et al. (2015). SKIP Confers Osmotic Tolerance during Salt Stress by Controlling Alternative Gene Splicing in Arabidopsis. Mol. Plant 8, 1038-1052. doi: 10.1016/j.molp.2015.01.011

Godoy Herz, M. A., Kubaczka, M. G., Brzyżek, G., Servi, L., Krzyszton, M., Simpson, C., et al. (2019). Light Regulates Plant Alternative Splicing through the Control of Transcriptional Elongation. Mol. Cell 73, 1066.e-1074.e. doi: 10.1016/j.molcel.2018.12.005

Gupta, S., Zink, D., Korn, B., Vingron, M., and Haas, S. (2004). Genome wide identification and classification of alternative splicing based on EST data. Bioinformatics 20, 2579-2585. doi: 10.1093/bioinformatics/bth288

Han, N., Ji, X. L., Du, Y. P., Xi, H., Zhao, X. J., and Zhai, H. (2017). Identification of a Novel Alternative Splicing Variant of VvPMA1 in Grape Root under Salinity. Front. Plant Sci. 8:605. doi: 10.3389/fpls.2017.00605

$\mathrm{Hu}$, J., Manduzio, S., and Kang, H. (2019). Epitranscriptomic RNA Methylation in Plant Development and Abiotic Stress Responses. Front. Plant Sci. 10:500. doi: 10.3389/fpls.2019.00500

Hu, Y., Mesihovic, A., Jiménez-Gómez, J. M., Roth, S., Gebhardt, P., Bublak, D., et al. (2019). Natural variation in HsfA2 pre-mRNA splicing is associated with changes in thermotolerance during tomato domestication. New Phytol. 225, 1297-1310. doi: 10.1111/nph.16221

Huang, C., Miki, D., Tang, K., Zhou, H., Zheng, Z., Zhu, J., et al. (2013). A Pre-mRNA-splicing factor is required for RNA-directed DNA methylation in Arabidopsis. PLoS Genet. 9:e1003779. doi: 10.1371/journal.pgen.1003779

Huertas, R., Catalá, R., Jimenez-Gomez, J., Castellano, M. M., Crevillén, P., Piñeiro, M., et al. (2019). Arabidopsis SME1 regulates plant development and response to abiotic stress by determining spliceosome activity specificity. Plant Cell 31, 537-554. doi: 10.1105/tpc.18.00689

Ibtissam, J., Reddy, A. S. N., Maria, K., Saurabh, C., Waqas, K., Byrne, L. J., et al. (2019). SURVEY AND SUMMARY Does co-transcriptional regulation of alternative splicing mediate plant stress responses? Nucleic Acids Res. 47, 2716-2726. doi: 10.1093/nar/gkz121

Jeong, S. (2017). SR Proteins: binders, Regulators, and Connectors of RNA. Mol. Cells 40, 1-9. doi: 10.14348/molcells.2017.2319

Jiang, J., Liu, X., Liu, C., Liu, G., Li, S., and Wang, L. (2017). Integrating Omics and Alternative Splicing Reveals Insights into Grape Response to High Temperature. Plant Physiol. 173:1502. doi: 10.1104/pp.16.01305

Kim, J. K., Lee, K. C., Chung, K. S., Lee, H. T., and Lee, J. H. (2020). Role of Arabidopsis Splicing factor SF1 in Temperature-Responsive Alternative Splicing of FLM pre-mRNA. Front. Plant Sci. 11:596354. doi: 10.3389/fpls.2020. 596354

Laloum, T., Martín, G., and Duque, P. (2018). Alternative Splicing Control of Abiotic Stress Responses. Trends Plant Sci. 23:140. doi: 10.1016/j.tplants.2017. 09.019

Lee, B., Kapoor, A., Zhu, J., and Zhu, J. (2006). STABILIZED1, a stress-upregulated nuclear protein, is required for pre-mRNA splicing, mRNA turnover, and stress tolerance in Arabidopsis. Plant Cell 18, 1736-1749. doi: 10.1105/tpc.106. 042184

Lee, J., Ryu, H., Chung, K., Posé, D., Kim, S., Ahn, J., et al. (2013). Regulation of temperature-responsive flowering by MADS-box transcription factor repressors. Science 342, 628-632. doi: 10.1126/science.1241097

Li, Y., Guo, Q., Liu, P., Huang, J., Zheng, C., Yan, K., et al. (2021). Dual roles of the serine/arginine-rich splicing factor SR45a in promoting and interacting with nuclear cap-binding complex to modulate the salt-stress response in Arabidopsis. New Phytol. 230, 641-655. doi: 10.1111/nph.17175

Li, Y., Mi, X., Zhao, S., Zhu, J., and Wei, C. (2020). Comprehensive profiling of alternative splicing landscape during cold acclimation in tea plant. BMC Genom. 21:65. doi: 10.1186/s12864-020-6491-6

Li, Z., Tang, J., Bassham, D. C., and Howell, S. H. (2021). Daily temperature cycles promote alternative splicing of RNAs encoding SR45a, a splicing regulator in maize. Plant Physiol. 186, 1318-1335.

Liu, J., Sun, N., Liu, M., Liu, J., Du, B., Wang, X., et al. (2013). An autoregulatory loop controlling Arabidopsis HsfA2 expression: role of heat shock-induced alternative splicing. Plant Physiol. 162, 512-521. doi: 10.1104/pp.112.205864

Luco, R. F., Allo, M., Schor, I. E., Kornblihtt, A. R., and Misteli, T. (2011). Epigenetics in Alternative Pre-mRNA Splicing. Cell 144, 16-26. doi: 10.1016/ j.cell.2010.11.056

Marcella, D., Willing, E. M., Szabo, E. X., Francisco-Mangilet, A. G., Droste-Borel, I., Macek, B., et al. (2018). The U1 snRNP subunit LUC7 modulates plant development and stress responses via regulation of alternative splicing. Plant Cell 30, 2838-2854. doi: 10.1105/tpc.18.00244

Marquez, Y., Brown, J., Simpson, C., Barta, A., and Kalyna, M. (2012). Transcriptome survey reveals increased complexity of the alternative splicing landscape in Arabidopsis. Genom. Res. 22, 1184-1195. doi: 10.1101/gr.134106. 111

Ner-Gaon, H., Halachmi, R., Savaldi-Goldstein, S., Rubin, E., and Fluhr, R. (2004). Intron retention is a major phenomenon in alternative splicing in Arabidopsis. Plant J. 39, 877-885. doi: 10.1111/j.1365-313X.2004.02172.x

Nibau, C., Gallemí, M., Dadarou, D., Doonan, J. H., and Cavallari, N. (2020). Thermo-Sensitive Alternative Splicing of FLOWERING LOCUS M Is Modulated by Cyclin-Dependent Kinase G2. Front. Plant Sci. 10:1680. doi: $10.3389 /$ fpls.2019.01680

Pajoro, A., Severing, E., Angenent, G. C., and Immink, R. (2017). Histone H3 lysine 36 methylation affects temperature-induced alternative splicing and flowering in plants. Genom. Biol. 18:102. doi: 10.1186/s13059-017-1235-x

Palusa, S. G., Ali, G. S., and Reddy, A. (2007). Alternative splicing of pre-mRNAs of Arabidopsis serine/arginine-rich proteins: regulation by hormones and stresses. Plant J. 49, 1091-1107. doi: 10.1111/j.1365-313X.2006. 03020.x

Pan, Q., Shai, O., Lee, L. J., Frey, B. J., and Blencowe, B. J. (2008). Deep surveying of alternative splicing complexity in the human transcriptome by high-throughput sequencing. Nat. Genet. 40, 1413-1415. doi: 10.1038/ng.259

Petrillo, E., Herz, M. G., Fuchs, A., Reifer, D., Fuller, J., Yanovsky, M. J., et al. (2014). A Chloroplast Retrograde Signal Regulates Nuclear Alternative Splicing. Science 344, 427-430. doi: 10.1126/science. 1250322

Posé, D., Verhage, L., Ott, F., Yant, L., Mathieu, J., Angenent, G., et al. (2013). Temperature-dependent regulation of flowering by antagonistic FLM variants. Nature 503, 414-417.

Punzo, P., Grillo, S., and Batelli, G. (2020). Alternative splicing in plant abiotic stress responses. Biochem. Soc. Trans. 48, 2117-2126. doi: 10.1042/ BST20200281

Sanchez, S. E., Petrillo, E., Beckwith, E. J., Zhang, X., and Yanovsky, M. J. (2010). A methyl transferase links the circadian clock to the regulation of alternative splicing. Nature 468, 112-116. doi: 10.1038/nature09470

Sanyal, S. K., Poonam, K., Harsha, S., Kanwaljeet, K., Jha, S. K., and Pandey, G. K. (2017). Alternative Splicing of CIPK3 Results in Distinct Target Selection to Propagate ABA Signaling in Arabidopsis. Front. Plant Sci. 8:1924. doi: 10.3389/ fpls.2017.01924

Seo, P. J., Kim, M. J., Ryu, J. Y., Jeong, E. Y., and Park, C. M. (2011). Two splice variants of the IDD14 transcription factor competitively form nonfunctional heterodimers which may regulate starch metabolism. Nat. Commun. 2:303. doi: $10.1038 /$ ncomms 1303

Sugliani, M., Brambilla, V., Clerkx, E. J. M., Koornneef, M., and Soppe, W. J. J. (2010). The Conserved Splicing Factor SUA Controls Alternative Splicing of 
the Developmental Regulator ABI3 in Arabidopsis. Plant Cell 22, 1936-1946. doi: 10.1105/tpc.110.074674

Sureshkumar, S., Dent, C., Seleznev, A., Tasset, C., and Balasubramanian, S. (2016). Nonsense-mediated mRNA decay modulates FLM-dependent thermosensory flowering response in Arabidopsis. Nat. Plant 2:16055. doi: 10.1038/nplants. 2016.55

Thatcher, S. R., Danilevskaya, O. N., Meng, X., Beatty, M., Zastrowhayes, G., Harris, C., et al. (2016). Genome-Wide Analysis of Alternative Splicing during Development and Drought Stress in Maize. Plant Physiol. 170:586. doi: 10.1104/ pp.15.01267

Wang, B., and Brendel, V. (2006). Genomewide comparative analysis of alternative splicing in plants. Proc. Natl. Acad. Sci. U.S.A. 103, 7175-7180. doi: 10.1073/ pnas.0602039103

Wang, L., Yang, T., Wang, B., Lin, Q., and Yu, F. (2020). RALF1-FERONIA complex affects splicing dynamics to modulate stress responses and growth in plants. Sci. Adv. 6:eaaz1622. doi: 10.1126/sciadv.aaz1622

Wang, M., Wang, P., Liang, F., Ye, Z., Li, J., Shen, C., et al. (2018). A global survey of alternative splicing in allopolyploid cotton: landscape, complexity and regulation. New Phytol. 217, 163-178. doi: 10.1111/nph.14762

Wang, X., Hu, L., Wang, X., Li, N., Xu, C., Gong, L., et al. (2016). DNA Methylation Affects Gene Alternative Splicing in Plants: an Example from Rice. Mol. Plant 9, 305-307. doi: 10.1016/j.molp.2015.09.016

Wang, Z., Ji, H., Yuan, B., Wang, S., Su, C., Yao, B., et al. (2015). ABA signalling is fine-tuned by antagonistic HAB1 variants. Nat. Commun. 6:8138. doi: 10.1038/ ncomms 9138

Wei, G., Liu, K., Shen, T., Shi, J., Liu, B., Han, M., et al. (2018). Position-specific intron retention is mediated by the histone methyltransferase SDG725. BMC Biol. 16:44. doi: 10.1186/s12915-018-0513-8

Wu, Z., Liang, J., Wang, C., Ding, L., Zhao, X., Yi, M., et al. (2019). Alternative Splicing Provides a Mechanism to Regulate LlHSFA3 Function in Response to Heat Stress in Lily. Plant Physiol. 181, 1651-1667. doi: 10.1104/pp.19.00839

Zhang, G., Guo, G., Hu, X., Yong, Z., Li, Q., Li, R., et al. (2010). Deep RNA sequencing at single base-pair resolution reveals high complexity of the rice transcriptome. Genom. Res. 20, 646-654. doi: 10.1101/gr.100677.109
Zhang, R., Calixto, C. P. G., Marquez, Y., Venhuizen, P., Tzioutziou, N. A., Guo, W., et al. (2017). A high quality Arabidopsis transcriptome for accurate transcriptlevel analysis of alternative splicing. Nucleic Acids Res. 45, 5061-5073. doi: 10.1093/nar/gkx267

Zhang, W., Du, B., Di, L., and Qi, X. (2014). Splicing factor SR34b mutation reduces cadmium tolerance in Arabidopsis by regulating iron-regulated transporter 1 gene. Biochem. Biophys. Res. Commun. 455, 312-317. doi: 10.1016/j.bbrc.2014. 11.017

Zhang, Z., Zhang, S., Zhang, Y., Wang, X., Li, D., Li, Q., et al. (2011). Arabidopsis floral initiator SKB1 confers high salt tolerance by regulating transcription and pre-mRNA splicing through altering histone H4R3 and small nuclear ribonucleoprotein LSM4 methylation. Plant Cell 23, 396-411. doi: 10.1105/tpc. 110.081356

Zhou, Y., Li, X., Guo, Q., Liu, P., Zheng, C., Yan, K., et al. (2021). Salt responsive alternative splicing of a RING finger E3 ligase modulates the salt stress tolerance by fine-tuning the balance of COP9 signalosome subunit 5A. PLoS Genet. 17:e1009898. doi: 10.1371/journal.pgen.1009898

Conflict of Interest: The authors declare that the research was conducted in the absence of any commercial or financial relationships that could be construed as a potential conflict of interest.

Publisher's Note: All claims expressed in this article are solely those of the authors and do not necessarily represent those of their affiliated organizations, or those of the publisher, the editors and the reviewers. Any product that may be evaluated in this article, or claim that may be made by its manufacturer, is not guaranteed or endorsed by the publisher.

Copyright (c) 2022 Liu, Guo, Xu, Liu and Yan. This is an open-access article distributed under the terms of the Creative Commons Attribution License (CC BY). The use, distribution or reproduction in other forums is permitted, provided the original author(s) and the copyright owner(s) are credited and that the original publication in this journal is cited, in accordance with accepted academic practice. No use, distribution or reproduction is permitted which does not comply with these terms. 